\title{
Ética e Desempenho Social das Organizações: um Modelo Teórico de Análise dos Fatores Culturais e Contextuais
}

\author{
Filipe Jorge Ribeiro de Almeida
}

\begin{abstract}
RESUMO
O despertar crescente dos gestores para a necessidade de respeitarem códigos de ética na sua atuação está relacionado com a percepção igualmente crescente, entre estes profissionais, de que, no longo prazo, a postura ética pode resultar em importantes vantagens competitivas decorrentes de uma imagem positiva projetada na comunidade e do aumento da satisfação e da melhoria do desempenho dos trabalhadores. No entanto, as escolhas dos gestores, perante dilemas éticos, são também influenciadas por fatores de ordem individual, não estratégicos, que podem ser decisivos no processo de tomada de decisão. Mais do que nunca, a compreensão desses fatores representa um instrumento essencial de construção de uma cultura favorável ao desenvolvimento ético da sociedade, e que garanta, ao mesmo tempo, a sustentabilidade desejável dos negócios. Este estudo pretende contribuir para o debate das questões éticas na prática empresarial, por meio da proposta de um modelo teórico, apoiado em hipóteses que exploram os fatores culturais, morais e contextuais que influenciam as opções de natureza social nas organizações.
\end{abstract}

Palavras-chave: ética; moral; cultura; desempenho social.

\begin{abstract}
The increasing awareness of managers concerning the need for them to respect codes of ethics in their jobs is closely related with their perception that in the long run an ethical posture can result in significant competitive advantages based on a positive external image of the corporation and higher levels of workers' satisfaction and performance. However, the choices managers made when facing ethical dilemas are influenced as well by individual factors, not strategical in nature, that can play an important role in the decision-making process. More than never, understanding these factors is a crucial step to develop a culture that enables ethical social development, and that assures, at the same time, the desirable business sustainability. This study aims to contribute for the debate on corporate ethics, presenting a theoretical model based on hypothesis that explore cultural, moral and contextual factors that can influence socially-based corporate practices.
\end{abstract}

Key words: ethics; moral; culture; corporate social performance. 


\section{INTRODUÇÃO}

As últimas décadas do século XX foram marcadas por transformações profundas em todo o mundo, estimuladas em grande medida pela democratização dos regimes políticos, pela abertura de fronteiras comerciais e por um desenvolvimento tecnológico sem precedentes. A magnitude destas mudanças tem impactos econômicos, sociais e culturais em escala mundial, cujos efeitos ainda não podem ser apreciados nem antecipados com exatidão. O fenômeno de globalização das economias e dos mercados arrasta uma tendência para a convergência de gostos, de normas, de comportamentos, de expectativas, de padrões de qualidade e de desempenho, constituindo uma pressão suplementar para todas as organizações e empresas, independentemente do nível em que operam - local, regional, nacional ou mundial.

O aumento significativo dos fluxos de comércio internacional, facilitados por tecnologias inovadoras de processamento de informação e de comunicação, tornou a dimensão ética da atividade empresarial uma das principais preocupações de gestores, políticos, pesquisadores e da sociedade em geral (Robertson, Crittenden, Brady, \& Hoffman, 2002). A adoção de uma conduta baseada em princípios morais que respeitem o ambiente e os valores da comunidade envolvente é uma exigência incontornável das sociedades contemporâneas que os responsáveis organizacionais não podem ignorar. A tolerância perante os abusos de poder ou a exploração inadequada de recursos é cada vez menor e a concorrência entre empresas passa também pelo alcance social e ambiental dos seus resultados e da sua atividade (Luce, Barber, \& Hillman, 2001). Por outro lado, à medida que as interações de gestores e executivos de diferentes países são mais freqüentes, a capacidade de compreender as diferenças de comportamento e as suas motivações morais é mais necessária para garantir o êxito dessas interações (Priem \& Shaffer, 2001).

A ética, enquanto disciplina teórica, estuda os códigos de valores que determinam o comportamento e influenciam a tomada de decisões num determinado contexto. Estes códigos têm por base um conjunto tendencialmente consensual de princípios morais, que determinam o que deve ou não deve ser feito em função do que é considerado certo ou errado por determinada comunidade. No ambiente organizacional e na gestão de empresas em particular, a ética estuda os códigos morais que orientam as decisões empresariais, na medida em que estas afetem 
as pessoas e a comunidade envolvente, partindo de um conjunto socialmente aceito de direitos e obrigações individuais e coletivos. As empresas consideradas éticas são geralmente aquelas cuja conduta é socialmente valorizada e cujas políticas se reconhecem sintonizadas com a moral vigente, subordinando as suas atividades e estratégias a uma reflexão ética prévia e agindo posteriormente de forma socialmente responsável.

Muitos pesquisadores têm-se dedicado a estudar a conduta ética dos gestores e os fatores que a influenciam no contexto empresarial, destacando-se, neste domínio, três níveis de explicação do comportamento ético: individual, organizacional e cultural. Um número elevado de estudos tem procurado explicar a postura ética dos executivos a partir de algumas das suas características individuais como o gênero (Deshpande, 1997; Elm, Kennedy, \& Lawton, 2001; Luthar, DiBattista, \& Gautschi, 1997; McDaniel, Schoeps, \& Lincourt, 2001), a idade (Ruegger \& King, 1992; Vitell, Lumpkin, \& Rawwas, 1991), o grau de instrução (Deshpande, 1997; Elm et al., 2001), as orientações filosóficas (Cherry \& Frederich, 2000; Deconinck \& Lewis, 1997; Singhapakdi, Vitell, \& Franke, 1999) ou os valores morais (Agle, Mitchell, \& Sonnenfeld, 1999; Carlson, Kacmar, \& Wadsworth, 2002; Glover, Bumpus, Logan, \& Ciesla, 1997; Ray \& Pauli, 2002). Em nível organizacional os determinantes mais estudados incluem o clima ético da organização (Deshpande, 1996; Frietzsche, 2000; Peterson, 2002; Vardy, 2001), o papel dos códigos de ética (Farrell, Cobin, \& Farrell, 2002; Hoivik, 2002; Schwartz, 2001; Wotruba, Chonko, \& Loe, 2001) e a estrutura do capital (Brower \& Shrader, 2000; Bucar \& Hisrich, 2001; Hornsby, Kuratko, Naffziger, LaFollette, \& Hodgetts, 1994; Johnson \& Greeing, 1999). Constituindo um interesse mais recente da comunidade científica, a investigação sobre a influência dos fatores de ordem cultural e social nos comportamentos éticos e nos sistemas de valores dos gestores tem produzido igualmente estudos empíricos de crescente profundidade e relevância (Jackson \& Artola, 1997; Priem \& Shaffer, 2001; Robertson et al., 2002; Tsui \& Windsor, 2001).

Para além do recurso a essas três ordens de fatores (individual, organizacional e cultural), considerados em geral estáveis, para explicar os comportamentos moralmente qualificáveis no contexto empresarial, alguns autores defendem ainda o caráter situacional das decisões éticas. Segundo esses autores, a decisão de um mesmo indivíduo pode ser diferente, consoante o contexto específico que envolva essa decisão (Hoffman, Couch, \& Lamont, 1998; Robertson et al., 2002), variando, por exemplo, de acordo com a posição que o indivíduo ocupa na empresa ou com a antecipação subjetiva que ele faça da reação pública à sua decisão. 
Por não exigirem um conhecimento técnico nem se regerem por leis de oferta e de procura, as decisões que envolvem um julgamento moral são as únicas que, em ambiente empresarial, estão sempre sujeitas a uma avaliação externa que pode condenar definitivamente ou amplificar significativamente o sucesso de qualquer iniciativa. O desempenho social da organização será, em última análise, a face visível do comportamento ético dos seus dirigentes, refletindo a boa ou a má prática empresarial que se refletirá, por seu lado, nos resultados financeiros e na própria sustentabilidade da atividade da empresa (Ruf, Muralidhar, Brown, Janney, \& Paul, 2001). Compreender os mecanismos que influenciam e explicam os comportamentos éticos é, portanto, um requisito indispensável para sobreviver num mercado global cada vez mais atento e mais exigente em relação às práticas empresariais.

Pretende-se neste artigo apresentar um modelo teórico explicativo do desempenho social das organizações a partir da análise dos seus determinantes culturais, organizacionais e individuais. Com base na revisão da literatura fundamental, propõe-se um modelo integrado, onde se relacionam os valores culturais, o desenvolvimento moral, o clima ético organizacional e o desempenho social, com o objetivo de destacar o papel central dos fatores não estratégicos nas opções de ação social das organizações. Com esta contribuição, pretende-se alargar o campo teórico da discussão atual sobre as motivações éticas da atuação empresarial, centrando a análise no papel decisivo que a visão subjetiva do dirigente organizacional pode ter na definição de estratégias socialmente responsáveis.

\section{Referencial Teórico}

\section{Fatores Culturais}

O comportamento social, os valores morais, a conduta individual e a reação perante o desconhecido são manifestações humanas que dependem em larga medida do contexto sociocultural, onde o indivíduo se insere e que este mantém como referência. O conceito de cultura está associado a este contexto sociocultual. Citado por Ogburn, Tyler definiu cultura como um espaço complexo, onde estão incluídos os conhecimentos, as crenças, as artes, os valores morais, as leis e os costumes, assim como qualquer outra capacidade ou hábito adquirido pelo indivíduo, enquanto membro de uma sociedade (Ogburn, 1964). Swidler sugere a noção de cultura como um conjunto de ferramentas (concretizadas em símbolos, histórias, rituais e visões do mundo) 
que os indivíduos podem utilizar em diferentes combinações para lidar com diferentes tipos de problema (Swidler, 1986). Num sentido mais lato, a cultura é normalmente encarada como um sistema de normas e de valores socialmente transmitido (Lynn, 1990). Hofstede, por seu lado, defende a idéia de que cada indivíduo transporta consigo uma programação mental constituída por componentes da cultura nacional onde está integrado, que foram transmitidas pela família, depois pela escola e mais tarde reforçadas pelas organizações onde trabalha. A cultura será esta programação coletiva que distingue um grupo do outro e que permite manter a ordem social ao tornar os comportamentos individuais previsíveis (Hofstede, 1980).

No seu amplamente citado e explorado estudo publicado em 1980, Hofstede conceitualizou a cultura através da sua decomposição em quatro dimensões (distância hierárquica, aversão à incerteza, individualismo e masculinidade $)^{(1)}$, apresentando um mapa cultural do mundo, como resultado de um vasto estudo empírico, que viria a servir de referência para a generalidade dos estudos posteriores sobre aspectos culturais dos indivíduos, das organizações e dos países. As dimensões propostas por Hofstede reúnem uma significativa aceitação entre os cientistas sociais e a sua validade tem sido repetidamente demonstrada em inúmeros trabalhos (Robertson et al., 2002). Neste mapa cultural do mundo é possível identificar grupos homogêneos de países, em cujo âmbito os perfis culturais são idênticos entre si em aspectos pertinentes para a análise das questões éticas. Apesar das dimensões de Hofstede serem habitualmente aplicadas ao nível nacional, alguns autores sugerem que elas também são identificáveis ao nível individual (Wagner, 1995; Triandis, Bontempo, Villareal, Asai, \& Lucca, 1988), considerando-as bons indicadores do comportamento e critério válido para distinguir pessoas.

No contexto organizacional, o indivíduo não está imune aos efeitos e às influências da cultura social. Um dos fatores que pode influenciar a forma como uma pessoa reage perante diferentes dilemas éticos ${ }^{(2)}$ é precisamente a sua perspectiva cultural (Robertson et al., 2002). Nos anos mais recentes, o interesse da comunidade científica pelo estudo do fenômeno ético a partir de uma abordagem cultural tem aumentado; no entanto, o número de trabalhos realizados sobre o tema ainda é reduzido (Jackson \& Artola, 1997) e os resultados globais ainda são inconclusivos (Priem \& Shaffer, 2001). Numa abordagem da ligação entre as referências culturais e a ética profissional, Tsui e Windsor estudaram a relação entre a teoria cultural de Hofstede e o desenvolvimento moral de auditores chineses e australianos, tendo encontrado suporte empírico para essa relação (Tsui \& Windsor, 2001). Outros autores têm desenvolvido estudos empíricos que corroboraram a existência de uma relação entre as dimensões culturais e a avaliação moral dos 
indivíduos perante dilemas éticos (Husted, Dozier, McMahon, \& Kattan, 1996; Jackson \& Artola, 1997; Priem \& Shaffer, 2001; Vitell, Nwachukwu, \& Barnes, 1993), mas a medida exacta desta relação ainda não é muito clara, dado existirem igualmente autores que não encontraram evidência empírica da sua existência (Lysonski \& Gaidis, 1991; Whipple \& Swords, 1992).

O relativismo cultural sugerido pelos estudos que estabelecem uma relação entre cultura e ética contraria a visão deontológica que defende um quadro estável de valores morais idênticos para todas as sociedades em todo o mundo. Segundo Moon e Woolliams, a dimensão ética da atividade empresarial tem especificidades culturais difíceis de evitar (Moon \& Woolliams, 2000) e os decisores devem estar preparados para compreender e aproveitar essas particularidades inerentes à condição social da vida humana.

\section{Desenvolvimento Moral Cognitivo}

As teorias atuais sobre o desenvolvimento moral dos indivíduos têm o seu fundamento nos trabalhos exploratórios de Jean Piaget sobre os processos de avaliação moral desenvolvidos pelas crianças. Piaget acrescentou um determinante cognitivo ao conceito de moralidade que, até então, era explicado com recurso exclusivo a determinantes ambientais (Piaget, 1932). Em 1969, o psicólogo Lawrence Kohlberg, baseado nas experiências e nos escritos de Piaget, formulou a teoria do desenvolvimento moral cognitivo (cognitive moral development), estudando especialmente a dimensão cognitiva do julgamento moral e a forma como este influencia o comportamento humano. Esta teoria tornou-se a base da pesquisa científica no campo do pensamento moral.

Kohlberg explorou os fatores que influenciam a percepção moral dos indivíduos desde a infância até à idade adulta e os processos de tomada de decisão que lhe estão associados. O modelo de Kohlberg baseia-se no conceito de justiça como fundamento da moralidade e sugere que um indivíduo progride através de um conjunto de fases seqüenciais, à medida que desenvolve as suas percepções morais (Kohlberg, 1969). Este processo cognitivo compreende três níveis de desenvolvimento moral - pré-convencional, convencional e pós-convencional -; cada um se subdivide em duas fases, também elas seqüenciais. A passagem de um nível para outro pressupõe uma progressão no desenvolvimento moral do indivíduo e uma mudança na forma de pensar que, em geral, não admite retrocessos. Em cada nível existem duas fases, representando a segunda uma forma mais avançada e organizada da primeira. Na Figura 1 é apresentado um resumo dos níveis de desenvolvimento moral. 
Figura 1: Fases de Desenvolvimento Moral

\section{NÍVEL PRÉ-CONVENCIONAL}

Fase 1 - Orientação para o Castigo e para a Obediência (Serei descoberto?)

Fase 2 - Orientação Relativista Instrumental (Que ganhos posso obter?)

\section{NÍVEL CONVENCIONAL}

Fase 3 - Orientação "Bom Moço/Boa Moça"

(Ser uma boa pessoa)

Fase 4 - Orientação para a Lei e para a Ordem

(As normas promovem o bem-estar social)

\section{NÍVEL PÓS-CONVENCIONAL}

Fase 5 - Orientação Normativa de Contrato Social

(Normas sociais aceitas por consenso alargado)

Fase 6 - Orientação para os Princípios Universais de Ética (Princípios éticos adotados independentemente dos valores sociais)

Fonte: adaptado de Elm e Nichols, 1993.

No primeiro nível de percepção moral - pré-convencional - os indivíduos tomam as decisões com base nas consequiências ou recompensas das suas ações, associando os conceitos de 'certo' e de 'errado' aos efeitos do seu comportamento e cedendo o seu julgamento ao exercício autoritário de poder. No nível convencional, a opinião dos grupos sociais envolventes passa a ter importância para a tomada de decisões que, na generalidade, visam satisfazer e obter a aprovação dos outros, verificando-se uma aderência a comportamentos, normas e valores aceitos pela sociedade em geral. Finalmente, à medida que o indivíduo atinge a maturidade moral, evolui para o nível pós-convencional, verificando-se um esforço por desenvolver padrões morais pessoais regulados por uma consciência crítica do mundo e independentes das normas estabelecidas por via da autoridade de qualquer outro agente social (Kohlberg, 1969, 1984). 
Apesar de alguns autores criticarem a rigidez da formulação teórica do modelo de Kohlberg (Elm \& Weber, 1994; Gilligan, 1977; Trevino, 1992), inúmeros estudos têm demonstrado ao longo dos anos a sua validade empírica (Blasi, 1980; Thoma, 1985; Weber \& Wasieleski, 2001), constituindo o referencial teórico mais divulgado entre os cientistas que estudam o desenvolvimento moral e os aspectos cognitivos do processo psicológico subjacente. No seguimento dos trabalhos de Kohlberg, James Rest (1979) sugeriu algumas variações ao modelo originalmente proposto, flexibilizando a sua articulação teórica e facilitando a operacionalização da pesquisa empírica sobre desenvolvimento moral. O modelo de Rest baseia-se no conceito de justiça social, atribuindo ao conceito de justiça, apresentado por Kohlberg como um valor individual, um sentido mais alargado, associado à cooperação social (Rest, 1979, 1986). No modelo de Rest, um indivíduo pode manifestar diferentes níveis de moralidade, consoante o contexto e as características do dilema ético, contrariando o sentido unidireccional da evolução moral proposto por Kohlberg que defende que cada pessoa se encontra, em cada momento, num determinado estádio de desenvolvimento moral, que se manifesta da mesma forma na resposta a qualquer circunstância que envolva um conflito de valores.

O quadro de valores morais de um indivíduo influencia e condiciona fortemente o seu comportamento. A visão moral do mundo de um dirigente é referencial importante para compreender algumas das suas decisões e das suas escolhas entre opções alternativas. Desta forma, assumindo a ligação entre valores morais e comportamento, pode estabelecer-se uma relação entre o nível de desenvolvimento moral de um dirigente e a orientação social das práticas organizacionais que ele define e influencia. A gestão ética e o desempenho social das organizações pode efetivamente constituir, ainda que parcialmente, um reflexo da maturidade moral dos seus dirigentes.

\section{Clima Ético Organizacional}

O clima organizacional é geralmente identificado como a atmosfera psicológica, social e humana que caracteriza a forma como as pessoas se relacionam entre si dentro da organização. A dimensão ética do clima organizacional foi originalmente conceitualizada por Victor e Cullen (1988). Estes autores definiram o clima ético organizacional como um conceito multifacetado composto pelas percepções partilhadas pelos membros de uma organização sobre quais são os comportamentos eticamente corretos e qual a forma como devem ser abordados os assuntos moralmente qualificáveis (Victor \& Cullen, 1988). Desta forma, o clima ético é afetado pelos diversos sistemas normativos da organização, tais como as políticas, os procedimentos, os esquemas remuneratórios e os sistemas de controlo (Barnett \& Vaicys, 2000). 
Wyld e Jones sugerem que a percepção de cada indivíduo sobre o clima ético da organização a que pertence desempenha um papel determinante nos processos de decisão individual (Wyld \& Jones, 1997). Wimbush argumenta, por seu lado, que, se os supervisores conseguem influenciar o clima organizacional, os gestores poderão influenciar o comportamento ético dos funcionários através do efeito das suas ações no clima ético de grupos de trabalho onde a atitude ética é inadequada (Wimbush \& Markham, 1997). Trevino defende que as pessoas procuram freqüentemente fora de si mesmas uma orientação para tomar decisões perante dilemas éticos, concluindo que as organizações poderão moderar a relação entre a avaliação cognitiva que os indivíduos fazem da realidade e o seu comportamento, através do incentivo do comportamento ético, do desenvolvimento de normas organizacionais e da responsabilização das hierarquias (Trevino, 1986). De fato, diversos estudos sugerem a existência de uma relação entre o clima ético e o comportamento individual (Deshpande, 1996; Frietzsche, 2000; Peterson, 2002), confirmando a pertinência do conceito e justificando-o como variável organizacional que ajuda a explicar as opções de natureza social que caracterizam a prática empresarial.

\section{Desempenho Social das Empresas}

A presença de preocupações sociais nas práticas empresariais pode ser encontrada em inúmeros exemplos e referências ao longo da história da humanidade. No entanto, apenas no século XX foram publicados os primeiros trabalhos formais sobre responsabilidade social. Em especial, o livro Social Responsibilities of the Businessman, de Howard Bowen (1953), representa o marco decisivo que dá início à era moderna da literatura sobre Responsabilidade Social das Empresas (RSE). No seu livro, Bowen considera que as empresas, devido à sua dimensão e proliferação crescentes, haviam adquirido uma influência alargada na estruturação e desenvolvimento das sociedades. Segundo o autor, este aumento de poder deveria ser acompanhado por um aumento da responsabilidade, constituindo portanto a RSE a obrigação de os empresários adotarem políticas e práticas adequadas aos objetivos e valores da sociedade (Bowen, 1953). Desde então, o conceito tem sido explorado e debatido por estudiosos, acadêmicos, empresários, políticos e cidadãos em todo o mundo, existindo já um corpo significativo de contribuições teóricas que têm promovido o desenvolvimento do conceito e que facilitam a sua compreensão. No entanto, apesar do debate e do reconhecimento da sua importância nas sociedades contemporâneas, a RSE não é um tema consensual, dadas as múltiplas definições e interpretações que tem suscitado e a complexidade das suas motivações e implicações. Depois de Bowen, vários autores acrescentaram dimensões, especificaram conteúdos e delimitaram fronteiras no estudo da RSE. Submetido 
a abordagens segundo critérios ideológicos, morais ou econômicos, o termo RSE adquiriu sentidos diversos que, na maioria dos casos, são complementares entre si. No contexto empresarial, a designação responsabilidade social visa destacar o papel central que as empresas representam, enquanto organizações humanas, na manutenção e desenvolvimento do bem-estar social. O conjunto de responsabilidades sociais de uma empresa engloba as ações e políticas de alcance alargado que visam contribuir para o equilíbrio social da comunidade envolvente, situando-se, no entanto, além das exigências específicas da finalidade que justifica a existência da própria organização. Segundo Ackerman, a responsabilidade social implica um acompanhamento permanente e uma avaliação sistemática das condições ambientais, centrando a análise nas necessidades dos diferentes stakeholders ligados à organização (Ackerman, 1975).

As políticas socialmente responsáveis e o respeito ativo por valores morais e sociais constituem os indicadores teóricos do desempenho social da empresa (DSE). Carroll defende que o DSE compreende três componentes: (1) o nível de responsabilidade social, (2) o comprometimento com causas sociais e (3) a filosofia subjacente à resposta das empresas perante problemas ou desafios de natureza social (Carroll, 1979). Wartick e Cochran concretizam a definição de DSE na observação de três dimensões da atividade empresarial: os princípios (valores que orientam as políticas de responsabilidade social), os processos (mecanismos utilizados para atuar socialmente) e as políticas sociais (Wartick \& Cochran, 1985). Donna Wood acrescenta aos princípios, aos processos e às políticas promovidas pela empresa, os resultados observáveis e os impactos sociais da sua ação (Wood, 1991).

O DSE está relacionado mais com os meios do que com os fins da atividade empresarial. O resultado financeiro, o volume de vendas, a quota de mercado ou a valorização do capital são indicadores que medem a concretização dos fins a que se destina a atividade da empresa. O DSE espelha a forma como a empresa, no desenrolar da sua atividade, utiliza os recursos disponíveis e promove o seu desenvolvimento, transfere meios para apoiar causas sociais, protege e respeita o ambiente e a comunidade envolvente (Miles, 1987). Destaca-se, por exemplo, no âmbito do DSE, o tratamento justo e responsável dos trabalhadores, as práticas transparentes, a limitação dos desperdícios e da produção de matérias poluentes ou os donativos e apoio a obras e iniciativas comunitárias de índole social, cultural ou desportiva. 


\section{Modelo Integrado do Processo Ético}

A ética, enquanto valor subjetivo da ação humana, está presente num número significativo de decisões cotidianas da atividade empresarial (Weber \& Wasieleski, 2001), tendo-se tornado um dos elementos centrais da avaliação do desempenho das empresas e dos seus responsáveis. Apesar da teoria exposta ser aplicável à generalidade das organizações, a proposta ganha especial relevância no contexto das empresas com fins lucrativos, sendo em seguida analisada para esse tipo particular de organização. Com base na revisão de literatura efetuada, propõe-se então um modelo teórico que integra os fatores individuais subjetivos - culturais e morais - que influenciam as práticas empresariais que afetam o seu desempenho social. Este modelo é centrado no papel do dirigente enquanto agente privilegiado de decisão, destacando o efeito dos valores culturais no seu desenvolvimento moral e no clima ético da organização que, por sua vez, influenciam a natureza do desempenho social empresarial. Apresenta-se em seguida o modelo provisoriamente designado de modelo integrado do processo ético, procurando assim sintetizar a dimensão da ética individual no processo de decisão que afeta as responsabilidades sociais das empresas:

\section{Figura 2: Modelo Integrado do Processo Ético Organizacional}

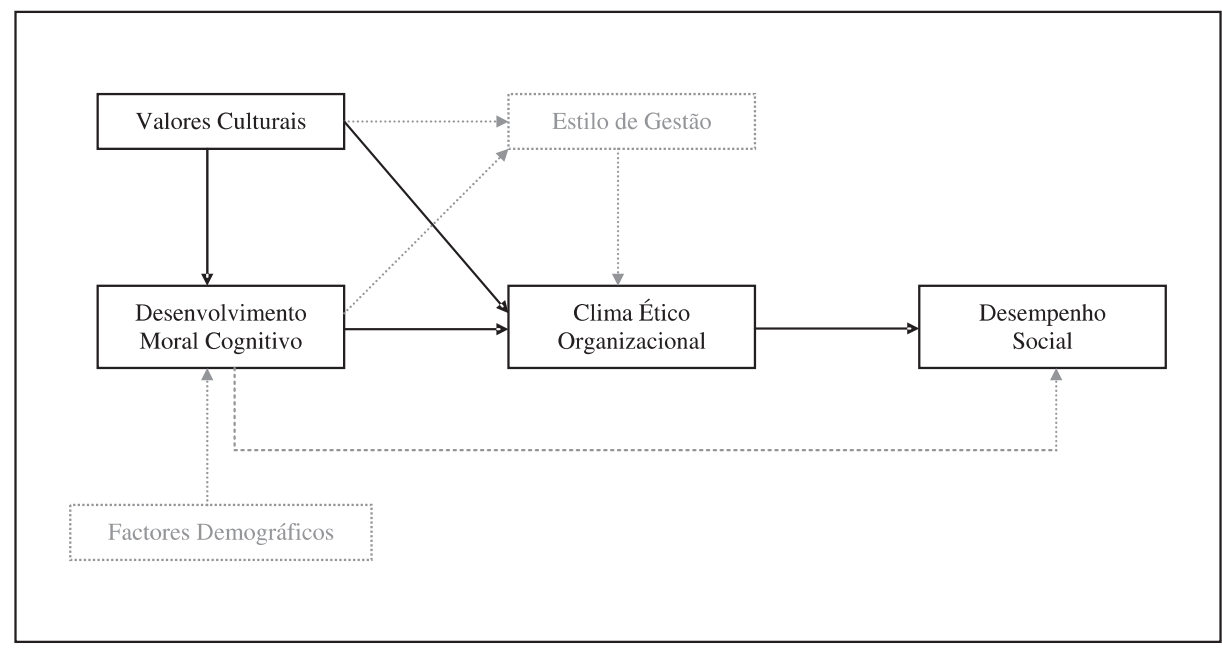

O modelo proposto visa reunir as principais variáveis que caracterizam o processo de decisão ética no contexto organizacional, integrando determinantes culturais, organizacionais e individuais. Trata-se de um modelo que explica parcialmente o desempenho social com recurso a variáveis contextuais e à percepção dos dirigentes organizacionais. No modelo, as relações entre Cultura, 
Desenvolvimento Moral, Clima Ético e Desempenho Social representam o eixo central, acrescentando ainda a influência secundária dos fatores demográficos e do estilo de gestão.

Apesar do seu sentido aparentemente determinista, não se pretende com este modelo explicar todo o processo de decisão ética nem estabelecer uma relação de causalidade única entre as variáveis. Pretende-se sim evidenciar o grau de correlação que existe entre as variáveis em causa, destacando a importância dos determinantes não estratégicos nas práticas de responsabilidade social das organizações. Os determinantes culturais e demográficos são as únicas variáveis independentes do modelo, subjacente ao qual existe um conjunto articulado de hipóteses de pesquisa. Estas hipóteses são desenvolvidas em seguida, pretendendose, através delas, explicitar com maior detalhe os fundamentos teóricos que justificam as relações propostas.

\section{Hipóteses do Modelo}

O modelo apresentado compreende um conjunto articulado de hipóteses subjacentes que, partindo do adequado enquadramento teórico, representam a principal contribuição intelectual da reflexão aqui proposta. Em seguida são apresentadas essas hipóteses, distinguindo uma hipótese central e a sua decomposição em diversas hipóteses básicas representantes das diversas relações entre as variáveis que constituem o modelo.

Hipótese Teórica: $\mathrm{O}$ contexto sociocultural e o perfil moral dos dirigentes organizacionais têm um impacto determinante no comportamento ético interno e externo da organização.

Hipótese Básica 1a (HB1a): Os dirigentes organizacionais que partilham referências socioculturais semelhantes apresentam um índice de desenvolvimento moral idêntico entre si.

Hipótese Básica 1b (HB1b): O contexto sociocultural exerce uma influência significativa no clima ético da organização.

Hipótese Básica 1c (HB1c): O contexto sociocultural exerce uma influência significativa no estilo de gestão e de liderança praticado na organização. 
Hipótese Básica 2a (HB2a): O desenvolvimento moral dos dirigentes organizacionais exerce uma influência positiva significativa no clima ético da organização.

Hipótese Básica 2b (HB2b): O desenvolvimento moral dos dirigentes organizacionais exerce uma influência significativa no estilo de gestão e de liderança praticado na organização.

Hipótese Básica 2c (HB2c): O desenvolvimento moral dos dirigentes organizacionais exerce uma influência positiva significativa no desempenho social da organização.

Hipótese Básica 3 (HB3): O clima ético organizacional exerce uma influência positiva significativa no desempenho social da organização.

Hipótese Básica 4 (HB4): $\quad$ O estilo de gestão e de liderança praticado na organização exerce uma influência significativa no clima ético organizacional.

Hipótese Básica 5 (HB5): O desenvolvimento moral dos dirigentes organizacionais é influenciado significativamente pelas suas características pessoais.

O DSE representa um fator de desenvolvimento organizacional, na medida em que as empresas com níveis elevados de DSE mantêm, à partida, relações saudáveis e de confiança com os diversos grupos de interesse com os quais se relacionam e dos quais dependem, permitindo assim reduzir os níveis de risco financeiro (Orlitzky \& Benjamin, 2001). A investigação sobre o DSE tem produzido poucos trabalhos que estudem o papel dos dirigentes organizacionais como determinantes do desempenho social (Thomas \& Simerly, 1995). A Hipótese Teórica deste estudo representa o problema central que se pretende esclarecer e tem subjacente a idéia de que o comportamento ético da organização é fortemente influenciado pela filosofia pessoal e pelos processos cognitivos de decisão dos seus dirigentes, apoiada nas idéias de Miles (1987) e Ackerman (1975).

A Hipótese HB1a representa uma das relações centrais propostas pelo modelo teórico e destaca a forte relação entre o contexto sociocultural e o índice de desenvolvimento moral dos dirigentes, baseando a sua formulação em estudos como os de Tsui e Windsor (2001), Husted et al. (1996) e Vitell (1993). As Hipóteses 
HB1b e HB1c estendem o impacto do contexto cultural ao clima ético da organização e ao estilo de direção que a caracteriza.

As Hipóteses HB2a, HB2b e HB2c referem-se à teoria de desenvolvimento moral cognitivo de Kohlberg como indicador-chave do índice moral dos dirigentes empresariais. Esta teoria representa a base teórica mais utilizada no estudo empírico da influência dos processos cognitivos de decisão no comportamento ético (Trevino, 1992; Tsui \& Windsor, 2001).

A Hipótese HB3 destaca a influência do clima ético organizacional na extensão e profundidade das políticas de natureza social conduzidas pela empresa. A formulação desta hipótese baseia-se na base alargada de estudos empíricos que relacionam o clima ético com o comportamento ético (Barnett \& Vaicys, 2000; Peterson, 2002).

A Hipótese HB4 introduz o estilo de gestão como variável influenciada pelo contexto cultural e pelo quadro ético de referência dos dirigentes, afetando, por sua vez, o clima ético organizacional. O estilo de gestão é ainda um determinante pouco estudado no âmbito das políticas e estratégias empresariais; no entanto, existem já alguns estudos que o destacam como uma variável central na explicação de decisões empresariais em geral (Almeida, 2001) e de opções éticas em particular (Pennino, 2002).

Finalmente, na seqüência dos resultados apresentados por um número significativo de estudos empíricos (Deshpande, 1997; Elm \& Nichols, 1993; Elm et al., 2001; Ruegger \& King, 1992; Thoma, 1985), a Hipótese HB5 estabelece uma relação causal entre as características pessoais do decisor como a idade, o gênero, o grau de instrução ou a função desempenhada, e o seu índice de desenvolvimento moral.

\section{Conclusão}

Os responsáveis organizacionais, especialmente aqueles que ocupam lugares de intervenção estratégica, estão freqüentemente expostos a pressões éticas consideráveis decorrentes dos impactos que as suas decisões podem ter nos trabalhadores, nos clientes, no mercado, na comunidade em geral ou no meio ambiente (Watson, 2003). As suas decisões e a sua atitude perante conflitos de interesse que envolvam valores morais influenciam o clima ético da organização, o comportamento dos trabalhadores e a forma como eles se comportam e se relacionam entre si e com a empresa (Bews \& Rossouw, 2002). A postura ética 
dos gestores e o clima ético da organização podem estimular ou reprimir o desenvolvimento de uma política social ativa, refletindo-se no desempenho real e percepcionado da empresa.

O objetivo deste ensaio teórico é contribuir para o debate em torno da ética empresarial e dos seus determinantes, enquanto tema que preocupa empresários e cidadãos em todo o mundo na atualidade. Ao estabelecer uma relação entre o contexto cultural, o desenvolvimento moral e o desempenho social da empresa, pretendeu-se destacar o papel do dirigente nas políticas sociais das empresas, reconhecendo o caráter situacional dos valores morais e sociais. A prática empresarial deve ser interpretada no seu contexto, constituindo o comportamento ético um reflexo do mesmo. Por outro lado, o modelo integrado de determinantes do desempenho social destaca a importância dos fatores não estratégicos que habitualmente presidem a uma parte significativa das decisões perante questões de natureza ética ou de intervenção social.

Subjacente ao modelo teórico, foram desenvolvidas hipóteses de pesquisa cuja validade pode e deve ser explorada em futuros estudos empíricos. Com a realização destes estudos, espera-se encontrar uma forte relação entre os referenciais culturais e os valores morais, assim como entre estes e as preocupações sociais reveladas pelas práticas empresariais. A riqueza dos resultados será reforçada se o modelo for aplicado em diferentes contextos culturais. As questões éticas, as problemáticas ambientais e as preocupações sociais são hoje temas centrais no debate político, social e empresarial. Este trabalho é certamente fruto dessas preocupações, ao mesmo tempo que pretende ser uma contribuição modesta mas determinada para a amplificação do debate urgente em torno destas temáticas.

\section{Artigo recebido em 19.10.2004. Aprovado em 01.12.2004.}

\section{Notas}

\footnotetext{
${ }^{1}$ A distância hierárquica respeita ao grau com que as desigualdades entre os indivíduos são aceites como naturais numa determinada sociedade. A aversão à incerteza descreve a atitude básica dos indivíduos face ao risco e a forma como reagem perante situações ambíguas. O individualismo e o seu oposto, o coletivismo, dizem respeito à forma como as pessoas se relacionam entre si e o perímetro das suas atenções afectivas. A masculinidade mede o grau com que uma dada comunidade valoriza atributos considerados masculinos, como a assertividade, o materialismo ou a competitividade.

${ }^{2}$ Segundo Robertson et al. (2002), um dilema ético é uma situação onde um indivíduo pode ver dois lados de um problema, sem que exista uma opção moral claramente certa ou errada que justifique uma decisão óbvia.
} 


\section{Referências Bibliográficas}

Ackerman, R. (1975).

The social challenge of business. Cambridge: Harvard Business Press.

Agle, B.,

Mtchell, R., \&

Sonnenfeld, J. (1999).

Who matters to CEOS? An investigation of stakeholders attributes and salience, corporate performance, and CEO values. Academy of Management Journal, 42(5), 507-525.

Almeida, F. (2001).

Organizações, pessoas e novas tecnologias. Coimbra: Quarteto Editora.

Barnett, T., \&

Vaicys, C. (2000).

The moderating effect of individuals' perceptions of ethical work climate on ethical judgements and behavioral intentions. Journal of Business Ethics, 27(4), 351-362.

Bews, N. F., \&

Rossouw, G. J. (2002).

A role for business ethics in facilitating trustworthiness. Journal of Business Ethics, 39(4), 377-390.

Blasi, A. (1980).

Bridging moral cognition and moral action: a critical review of the literature. Psychological Bulletin, 88(1), 1-45.

Bowen, H. (1953).

Social responsibilities of the businessman. New York: Harper \& Row.
Brower, H. H., \&

Shrader, C. B. (2000).

Moral reasoning and ethical climate: not-for-profit vs. For-profit Board of Directors. Journal of Business Ethics, 26(2), 147-167.

Bucar, B., \&

Hisrich, R. (2001).

Ethics of business managers vs. entrepreneurs. Journal of Developmental Entrepreneurship, 6(1), 59-82.

Carlson, D.,

Kacmar, K. M., \&

Wadsworth, L. (2002).

The impact of moral intensity dimensions on ethical decision making: assessing the relevance of orientation. Journal of Managerial Studies, 14(1), 15-30.

Carroll, A. (1979).

A three-dimensional conceptual model of corporate social performance. Academy of Management Review, 4(4), 497-505.

Cherry, J., \&

Frederich, J. (2000).

An empirical investigation of locus of control and the structure of moral reasoning: examining the ethical decision-making process of sales managers. The Journal of Personal Selling \& Sales Management, 20(3), 173-188. 
Deconinck, J., \&

Lewis, W. (1997).

The influence of deontological and teleological considerations and ethical climate on sales managers' intentions to reward or punish sales force behavior. Journal of Business Ethics, 16(5), 497-506.

Deshpande, S. (1996).

Ethical climate and the link between success and ethical behavior: an empirical investigation of non-profit organization. Journal of Business Ethics, 15(3), 315-320.

Deshpande, S. (1997).

Managers perception of proper ethical conduct: the effect of sex, age and level of education. Journal of Business Ethics, 16(1), 79-85.

Elm, D.,

Kennedy, E., \&

Lawton, L. (2001).

Determinants of moral reasoning: sex role orientation, gender and academic factors. Business and Society, 40(3), 241-265.

Elm, D., \&

Nichols, M. (1993).

An investigation of the moral reasoning of managers. Journal of Business Ethics, 12(11), 817-833.

Elm, D., \&

Weber, J. (1994).

Measuring moral judgment: the moral judgment interview or the defining issues test. Journal of Business Ethics, 13(5), 341-355.
Farrell, B.,

Cobbin, D., \&

Farrell, H. (2002).

Codes of ethics - their evolution, development and other controversies. Journal of Management Development, 21(2), 152-163.

Frietzsche, D. (2000).

Ethical climates and the ethical dimensions of decision making. Journal of Business Ethics, 24(2), 125-140.

Gilligan, C. (1977).

In a different voice: women's conception of the self and of morality. Harvard Educational Review, 47(4), 481-517.

Glover, S.,

Bumpus, M.,

Logan, J., \&

Ciesla, J. (1997).

Re-examining the influence of individual values on ethical decision making. Journal of Business Ethics, 16(12-13), 1319-1329.

Hoffman, J.,

Couch, G., \&

Lamont, B. (1998).

The effect of firm profit versus personal economic well being on the level of ethical responses given by managers. Journal of Business Ethics, 17(3), 239-244.

Hofstede, G. (1980).

Culture's consequences. London: SAGE Publications.

Hoivik, H. (2002).

Professional ethics - a managerial opportunity in emerging organizations. Journal of Business Ethics, 39(1-2), 3-11. 
Hornsby, J.,

Kuratko, D.,

Naffziger, D.,

LaFollette, W., \&

Hodgetts, R. (1994).

Ethical perceptions of small-business owners: a factor analytic study. Journal of Small Business Management, 32(4), 9-16.

Husted, B.,

Dozier, J.,

McMahon, T., \&

Kattan, M. (1996).

Impact of cross-national carriers of business ethics on attitudes about questionable practices and form of moral reasoning. Journal of International Business Studies, 27(2), 391-411.

Jackson, T., \&

Artola, M. (1997).

Ethical beliefs and management behaviour: a cross-cultural comparison. Journal of Business Ethics, 16(11), 1163-1173.

Johnson, R., \&

Greeing, D. (1999).

Effects of corporate governance and institutional ownership types on corporate social performance. Academy of Management Journal, 42(5), 564-576.

Kohlberg, L. (1969).

Stage and sequence: the cognitivedevelopmental approach to socialization. In D. Goslin (Org.). Handbook of socialization theory and research (pp 347-480). New York: Rand McNally.
Kohlberg, L. (1984).

The philosophy of moral development. New York: Harper \& Row.

Luce, R.,

Barber, A., \&

Hillman, A. (2001).

Good deeds and misdeeds: a mediated model of the effect of corporate social performance on organizational attractiveness. Business and Society, 40(4), 397-415.

Luthar, H.,

DiBattista, R., \&

Gautschi, T. (1997).

Perceptions of what the ethical climate is and what It should be: the role of gender, academic status, and ethical education. Journal of Business Ethics, 16(2), 205-217.

Lynn, L. H. (1990).

Technology and organizations: a cross-national analysis. In P. S. Goodman \& L.S. Sproull (Orgs.). Technology and Organizations (pp. 174-199). Oxford: Jossey-Bass Publishers.

Lysonski, S., \&

Gaidis, W. (1991).

A cross-national comparison of ethics of business students. Journal of Business Ethics, 10(2), 141-150.

McDaniel, C.,

Schoeps, N., \&

Lincourt, J. (2001).

Organizational ethics: perceptions of employees by gender. Journal of Business Ethics, 33(3), 245-256. 
Miles, R. (1987).

Marketing the corporate social environment. Englewood Cliffs: Prentice-Hall.

Moon, C., \&

Woolliams, P. (2000).

Managing cross-cultural business ethics. Journal of Business Ethics, 27(1-2), 105-115.

Ogburn, W. F. (1964).

On culture and social change. Chicago: University of Chicago Press.

Orlitzky, M., \&

Benjamin, J. (2001).

Corporate social performance and firm risk: a meta-analytic review. Business and Society, 40(4), 369-396.

Pennino, C. M. (2002).

Is decision style related to moral development among managers in the U.S.? Journal of Business Ethics, 41(4), 337-347.

Peterson, D. (2002).

The relationship between unethical behavior and the dimensions of the ethical climate questionnaire. Journal of Business Ethics, 41(4), 313-326.

Piaget, J. (1932).

The moral judgment of the child. New York: Free Press.

Priem, R., \&

Shaffer, M. (2001).

Resolving moral dilemmas in business: a multicountry study. Business and Society, 40(2), 197-219.

Ray, D., \&

Pauli, K. (2002).

The role of moral intensity in ethical decision making. Business and Society, 41(1), 84-117.

Rest, J. (1979).

Development in judging moral issues. Minneapolis: University of Minnesota Press.

Rest, J. (1986).

Moral development: advances in research and theory. New York: Praeger Press.

Robertson, C. J., Crittenden, W., Brady, M. K., \& Hoffman, J. J. (2002).

Situational ethics across borders: a multicultural examination. Journal of Business Ethics, 38(4), 327-338.

Ruegger, D., \&

King, E. W. (1992).

A study of the effect of age and gender upon student business ethics. Journal of Business Ethics, 11(3), 179186.

Ruf, B.,

Muralidhar, K.,

Brown, R., Janney, J., \&

Paul, K. (2001).

Empirical investigation of the relationship between change in corporate social performance and financial performance: a stakeholder theory perspective. Journal of Business Ethics, 32(2), 143-156.

Schwartz, M. (2001).

The nature of relationship between corporate codes of ethics and behavior. Journal of Business Ethics, 32(3), 247-262. 
Singhapakdi, A.,

Vitell, S., \&

Franke, G. (1999).

Antecedents, consequences and mediating effects of perceived moral intensity and personal moral philosophies. Academy of Marketing Science Journal, 27(1), 19-35.

Swidler, A. (1986).

Culture in action: symbols and strategies. American Sociological Review, 51(2), 273-286.

Thoma, S. (1985).

Estimating gender differences in the comprehension and preference of moral issues. Development Review, 6(2), 165-180.

Thomas, A., \&

Simerly, R. (1995).

Internal determinants of corporate social performance: the role of top managers. Academy of Management Journal, (Best Papers Proceedings), pp. 411-415.

Trevino, L. (1986).

Ethical decision making in organizations: a person-situation interactionist model. Academy of Management Review, 11(3), 601-617.

Trevino, L. (1992).

Moral reasoning and business ethics: implications for research, education and management. Journal of Business Ethics, 11(5-6), 445-459.

Triandis, H.,

Bontempo, R.,

Villareal, M.,

Asai, M., \&

Lucca, N. (1988).

Individualism and collectivism: cross- cultural perspectives on self-ingroup relationships. Journal of Personality and Social Psychology, 54(2), 323338.

Tsui, J., \&

Windsor, C. (2001).

Some cross-cultural evidence on ethical reasoning. Journal of Business Ethics, 31(2), 143-150.

Vardy, Y. (2001).

Effects of organizational and ethical climates on misconduct at work. Journal of Business Ethics, 29(4), 325337.

Victor, B., \&

Cullen, J. (1988).

The organizational bases of ethical work climate. Administrative Science Quarterly, 33(1), 101-125.

Vitell, S.,

Lumpkin, J., \&

Rawwas, M. (1991).

Consumer ethics: an investigation of the ethical beliefs of elderly consumers. Journal of Business Ethics, 10(5), 365-375.

Vitell, S.,

Nwachukwu, S., \&

Barnes, J. (1993).

The influence of culture on ethical decision-making: an application of Hofstede's Typology. Journal of Business Ethics, 12(10), 753-760.

Wagner, J. (1995).

Studies of individualism-collectivism: effects on cooperation in groups. Academy of Management Journal, 38(1), 152-172. 
Wartick, S., \&

Cochran, P. (1985).

The evolution of the corporate social performance model. Academy of Management Review, 10(4), 758-769.

Watson, T. (2003).

Ethical choice in managerial work: the scope for moral choices in an ethically irrational world. Human Relations, 56(2), 167-185.

Weber, J., \&

Wasieleski, D. (2001).

Investigating influences on managers moral reasoning. Business and Society, 40(1), 79-111.

Whipple, T., \&

Swords, D. (1992).

Business ethics judgments: a crosscultural comparison. Journal of Business Ethics, 11(9), 671-678.
Wimbush, J., \&

Markham, S. (1997).

An empirical examination of the relationship between ethical climate and ethical behavior from multiple levels of analysis. Journal of Business Ethics, 16(16), 1705-1716.

Wood, D. (1991).

Corporate social performance revisited. Academy of Management Review, 16(4), 691-718.

Wotruba, T., Chonko, L., \& Loe, T. (2001).

Impact of ethics code familiarity on manager behavior. Journal of Business Ethics, 33(1), 59-69.

Wyld, D., \& Jones, C. (1997).

The Importance of context: the ethical work climate construct and models of ethical decision making - an agenda for research. Journal of Business Ethics, 16(4), 465-472. 